\title{
Ivabradine preserves dynamic sympathetic control of heart rate despite inducing significant bradycardia in rats
}

\author{
Toru Kawada ${ }^{1}$ ( ) Shuji Shimizu ${ }^{1} \cdot$ Kazunori Uemura $^{1} \cdot$ Yohsuke Hayama $^{1} \cdot$ Hiromi Yamamoto $^{2} \cdot$ Toshiaki Shishido $^{3}$. \\ Takuya Nishikawa ${ }^{1}$ Masaru Sugimachi ${ }^{1}$
}

Received: 2 August 2018 / Accepted: 28 August 2018 / Published online: 6 September 2018

(C) The Physiological Society of Japan and Springer Japan KK, part of Springer Nature 2018

\begin{abstract}
Ivabradine is a selective bradycardic agent that inhibits hyperpolarization-activated cyclic nucleotide-gated (HCN) channels. HCN channels play a key role in mediating the positive chronotropic response to sympathetic nerve stimulation (SNS). We examined whether ivabradine would interfere with dynamic sympathetic control of heart rate (HR). The effect of intravenous ivabradine $(2 \mathrm{mg} / \mathrm{kg}, n=7)$ or metoprolol $(10 \mathrm{mg} / \mathrm{kg}, n=6)$ on the transfer function from SNS to HR was examined in anesthetized rats. Ivabradine preserved the asymptotic dynamic gain of the HR transfer function and nearly doubled the asymptotic dynamic gain of the transfer function from SNS to the R-R interval. In contrast, metoprolol abolished dynamic sympathetic control of HR. Preserved dynamic sympathetic control of HR, with coexisting bradycardia, may contribute to some of the beneficial effects of ivabradine previously reported in clinical application.
\end{abstract}

Keywords Metoprolol $\cdot$ Transfer function $\cdot$ Sympathetic stimulation

\section{Introduction}

Sympathetic nerve stimulation (SNS) increases heart rate $(\mathrm{HR})$ via $\beta_{1}$-adrenergic mechanism. Norepinephrine released from sympathetic nerve terminals binds to $\beta_{1^{-}}$ adrenergic receptors on sinoatrial nodal cells, which in turn act on stimulatory $\mathrm{G}$ protein and activate adenylyl cyclase to increase the intracellular concentration of cyclic adenosine monophosphate (cAMP). As a second messenger, cAMP acts downstream on a variety of effectors, including protein kinase $\mathrm{A}$ (PKA) and hyperpolarization-activated cyclic nucleotide-gated $(\mathrm{HCN})$ channels. PKA affects intracellular $\mathrm{Ca}^{2+}$ dynamics to drive the spontaneous beating of pacemaker cells [1]. HCN channels contribute to the generation of the funny current $\left(I_{\mathrm{f}}\right)$ during diastolic depolarization. Activation of $\mathrm{HCN}$ channels increases the rate of

Toru Kawada

torukawa@ncvc.go.jp

1 Department of Cardiovascular Dynamics, National Cerebral and Cardiovascular Center, Osaka 565-8565, Japan

2 Division of Cardiology, Department of Medicine, Faculty of Medicine, Kindai University, Osaka 589-8511, Japan

3 Department of Research Promotion, National Cerebral and Cardiovascular Center, Osaka 565-8565, Japan the diastolic depolarization, resulting in a shortening of the $\mathrm{R}-\mathrm{R}$ interval (RRI). While cAMP directly activates HCN channels, PKA may also modulate HCN channel activation [2]. A selective bradycardic agent ivabradine reduces HR by inhibiting HCN channels [3]. It has been shown that elevated HR is an independent risk factor for cardiovascular disease [4]. In a clinical trial (SHIFT study) HR reduction with ivabradine improved outcomes in patients with chronic heart failure whose baseline HR values were 70 beats/min (bpm) or higher [5].

There is a possibility that inhibition of HCN channels by ivabradine attenuates sympathetic HR control because $\mathrm{HCN}$ channels mediate the positive chronotropic effect of $\beta_{1}$-adrenergic stimulation [3]. However, intravenous ivabradine does not attenuate the HR response range observed during baroreceptor static pressure changes, despite inducing significant bradycardia [6]. In humans, ivabradine reduces HR, but autonomic HR regulation is maintained [7]. What remains unknown is whether ivabradine significantly modifies dynamic sympathetic control of HR. The dynamic characteristics of a given system are important because the response speed of the system can be different depending on the dynamic characteristics even when the static response is the same. For instance, an increase in mean stimulation frequency of SNS and an administration of a neuronal 
norepinephrine uptake blocker desipramine both decrease the steady-state gain of the HR response to SNS [8]. However, the natural frequency associated with the low-pass characteristics of the HR response is decreased only in the desipramine protocol, suggesting that the removal rate of norepinephrine at the neuroeffector junction is important in determining the speed of the HR response to SNS. Such information could not be obtained by observing the static HR response to SNS alone. Elucidating the dynamic characteristics of the HR response to SNS is essential if we are to understand drug-related effects on HR because HR is controlled dynamically by the autonomic nervous system during daily activities. The present study aimed to examine the effects of ivabradine on dynamic sympathetic control of HR. In comparison, we also examined the effects of metoprolol, a $\beta_{1}$-adrenergic blocker. We used a transfer function analysis to quantify the dynamic sympathetic control of HR over a wide frequency range of physiological interest [9-11].

\section{Materials and methods}

Animals were cared for in strict accordance with the Guiding Principles for the Care and Use of Animals in the Field of Physiological Sciences, which has been approved by the Physiological Society of Japan. The Animal Subjects Committee at the National Cerebral and Cardiovascular Center reviewed and approved the experimental protocols.

\section{Surgical preparation}

Thirteen male Wistar-Kyoto rats (345-410 g body weight) were anesthetized by intraperitoneal injection $(2 \mathrm{ml} / \mathrm{kg})$ of a mixture of urethane $(250 \mathrm{mg} / \mathrm{ml})$ and $\alpha$-chloralose $(40 \mathrm{mg} /$ $\mathrm{kg}$ ). The rat was mechanically ventilated using room air with added oxygen. The anesthetic mixture was diluted to 18 -fold with physiological saline and administered continuously $(2-3 \mathrm{ml} / \mathrm{kg}$ ) through a catheter (PE-50, Becton-Dickinson and Company, Sparks, MD, USA) inserted into the right femoral vein. Another venous catheter (PE-50) was inserted into the left femoral vein for administration of ivabradine or metoprolol. Arterial pressure (AP) was measured through a catheter (PE-50) inserted into the right femoral artery. The instantaneous HR was measured from a body surface electrocardiogram (ECG) via a cardiotachometer (AT-601G, Nihon-Kohden, Japan). The RRI was calculated offline from the $1-\mathrm{kHz}$ sampled ECG data. The position of the $\mathrm{R}$ wave was determined by fitting a quadratic equation to consecutive three data points around the peak of the $\mathrm{R}$ wave. The results of the present study were not different between the analyses using the tachometer-derived HR and the HR calculated as $60,000 /$ RRI. Body temperature was maintained at around $38^{\circ} \mathrm{C}$ using a heating pad and a lamp.
Bilateral carotid sinus baroreceptor regions were isolated from the systemic circulation [12,13], and intracarotid sinus pressure was fixed at a prevailing AP level. The resultant carotid sinus pressure was $98.1 \pm 3.7 \mathrm{mmHg}$ across animals (mean $\pm \mathrm{SE}, n=13$ ). The bilateral vagal and aortic depressor nerves were sectioned at the neck. The bilateral cervical sympathetic nerves were sectioned, and a pair of stainlesssteel wire electrodes (AS633, Cooner Wire, Chatsworth, CA, USA) was attached to the sectioned right cervical sympathetic nerve for efferent SNS [11]. Silicone glue (KwikSil, World Precision Instruments, Sarasota, FL, USA) was used to secure the electrodes and nerve. Since we only cut the bilateral cervical sympathetic nerves, the sympathetic nerves directed to the heart, originating from the thoracic spinal cord, remained intact. However, baroreflex-mediated changes in the sympathetic drive were minimized by fixing the intracarotid sinus pressure and by sectioning the aortic depressor nerves.

\section{Protocols}

After the surgical preparation was completed, a settling period of at least $30 \mathrm{~min}$ was allowed before starting the protocol. The right cervical sympathetic nerve was stimulated dynamically with binary white noise for $12 \mathrm{~min}$. The switching interval of the binary white noise was $1000 \mathrm{~ms}$, which yielded relatively flat input power spectra up to approximately $0.5 \mathrm{~Hz}$. The stimulation frequency, amplitude, and pulse duration were set at $5 \mathrm{~Hz}, 10 \mathrm{~V}$, and $100 \mu \mathrm{s}$, respectively. Next, ivabradine (ivabradine hydrochloride, Tokyo Chemical Industry, Japan, dissolved in physiological saline at $2 \mathrm{mg} / \mathrm{ml}$, injected at $2 \mathrm{mg} / \mathrm{kg}, n=7$ ) or metoprolol (metoprolol tartrate, Tokyo Chemical Industry, Japan, dissolved in physiological saline at $10 \mathrm{mg} / \mathrm{ml}$, injected at $10 \mathrm{mg} / \mathrm{kg}, n=6$ ) was administered intravenously. The dose of ivabradine $(2 \mathrm{mg} / \mathrm{kg}$ ) was adopted from a previous study in conscious rats [14] and was approximately twice the effective dose $50\left(\mathrm{ED}_{50}\right)$ for the bradycardic effect in rats [15]. The dose of metoprolol $(10 \mathrm{mg} / \mathrm{kg})$ was chosen based on a previous study [16] so as to attain full sympathetic blockade for more than $30 \mathrm{~min}$. After a 10 -min stabilization period, the 12-min random SNS was repeated. The sequence of the binary white noise was identical in each rat but differed across rats.

\section{Data analysis}

Data were recorded at $1000 \mathrm{~Hz}$ using an analog-to-digital converter. The data analysis began $2 \mathrm{~min}$ after the onset of the random SNS. The data were resampled at $10 \mathrm{~Hz}$ and divided into ten half-overlapping segments with a length of 1024 points (102.4 s). In each segment, we removed the linear trend and applied a Hanning window. Fourier 
transformations of SNS and HR signals were ensembleaveraged over the ten segments to obtain the power spectra of the input $\left[S_{\mathrm{SNS} \cdot \mathrm{SNS}}(f)\right]$ and output $\left[S_{\mathrm{HR} \cdot \mathrm{HR}}(f)\right]$ and cross spectra between the input and output $\left[S_{\mathrm{HR} \text {.SNS }}(f)\right]$. The transfer function from SNS to HR was calculated using the following equation [17].

$H(f)=\frac{S_{\mathrm{HR} \cdot \mathrm{SNS}}(f)}{S_{\mathrm{SNS} \cdot \mathrm{SNS}}(f)}$.

The magnitude-squared coherence function was also calculated as an index of linear dependence between SNS and HR in the frequency domain [17]:

$\operatorname{Coh}(f)=\frac{\left|S_{\mathrm{HR} \cdot \mathrm{SNS}}(f)\right|^{2}}{S_{\mathrm{SNS} \cdot \mathrm{SNS}}(f) S_{\mathrm{HR} \cdot \mathrm{HR}}(f)}$.

The step response of HR to SNS was calculated from the time integral of the inverse Fourier transformation of the estimated transfer function, which would assist intuitive understanding of the dynamic characteristics of the HR response to SNS.

In the ivabradine protocol, the estimated transfer function from SNS to HR approximated a second-order low-pass filter with pure dead time $[8,10,11]$ :

$H(f)=\frac{K}{1+2 \zeta \frac{f}{f_{N}} j+\left(\frac{f}{f_{N}} j\right)^{2}} \mathrm{e}^{-2 \pi f L j}$,

where $f$ and $j$ denote frequency and imaginary units, respectively; $K($ in $\mathrm{bpm} / \mathrm{Hz})$ is the asymptotic value of dynamic gain as the frequency tends to zero; $f_{\mathrm{N}}(\mathrm{in} \mathrm{Hz})$ is the natural frequency of the second-order low-pass filter; $\zeta$ is the damping ratio (no units); and $L$ (in s) is the pure dead time. The damping ratio determines the system's behavior as underdamped $(0<\zeta<1)$, critically damped $(\zeta=1)$, or overdamped $(\zeta>1)$.

In the metoprolol protocol, the HR response to SNS was significantly attenuated after metoprolol, and the model transfer function (Eq. 3) was not able to properly describe the estimated transfer function. Hence, only dynamic gain values at the lowest frequency $\left(G_{0.01}\right)$ were used to represent the transfer function.

\section{Statistical analysis}

Data are presented as means and SE values. The pre-stimulation $\mathrm{HR}$ and AP were calculated by averaging HR and AP values for $1 \mathrm{~min}$, just before onset of random SNS. The mean $\mathrm{HR}$ and AP during SNS were calculated by averaging the HR and AP values over a period of the transfer function analysis $(563.2 \mathrm{~s})$. The effects of the test drug and SNS on HR and AP were examined using a two-way repeated-measures analysis of variance (ANOVA) [18]. The magnitude of the sympathetic HR responses (the difference between the prestimulation HR and the mean HR during SNS), before and after drug administration, were compared using a paired $t$ test.

The steady-state step response $\left(S_{50}\right.$, in $\left.\mathrm{bpm} / \mathrm{Hz}\right)$ was derived from an average of the last $10 \mathrm{~s}$ of the estimated step response. The $80 \%$ rise time $\left(T_{80 \%}\right.$, in s) was determined as the time at which the step response reached $80 \%$ of $S_{50}$. Note that $S_{50}$ and $T_{80 \%}$ are not parameters of the model transfer function (Eq. 3) and thus provide the information independently of model selection. The effects of the drug on the parameters of the step response and the transfer function were examined using paired $t$ tests. Averaged coherence values were calculated in low-frequency $(0.01-0.1 \mathrm{~Hz})$ and high-frequency $(0.1-0.5 \mathrm{~Hz})$ ranges and compared before and after drug administration using the bootstrap method, which does not require any assumption on the distribution of a given statistical quantity [19].

The bradycardic effect (the magnitude of the pre-stimulation HR reduction) was compared between ivabradine and metoprolol using an unpaired $t$ test. To examine whether the reciprocal relationship between the RRI and HR affected the results, the RRI data were likewise analyzed. For all statistical analyses, the differences were considered significant at $P<0.05$.

\section{Results}

Representative recordings of SNS, HR, RRI, and AP obtained from on animal in the ivabradine protocol are shown in Fig. 1. The first random SNS increased the mean HR and changed HR dynamically under the control conditions. Intravenous administration of ivabradine gradually reduced HR. AP decreased just after ivabradine administration and remained slightly decreased until the second SNS in this animal. The second random SNS increased the mean HR and changed HR dynamically. The magnitude of the dynamic HR response after ivabradine was similar to that observed before ivabradine. RRI showed changes reciprocal to HR. The magnitude of the dynamic RRI response to the random SNS seemed to increase after ivabradine. Averaged values of HR, RRI, and AP are depicted in Fig. 2. The effects of ivabradine and SNS on HR were statistically significant, whereas the interaction effect was insignificant. Ivabradine did not significantly affect SNS-induced increase in mean HR (control: $59.8 \pm 12.0 \mathrm{bpm}$ vs. ivabradine: $54.3 \pm 12.1 \mathrm{bpm}, P=0.164)$. The effects of ivabradine and SNS on RRI were also statistically significant without a significant interaction effect. However, ivabradine augmented SNS-induced reductions in mean RRI from $-21.1 \pm 3.8$ to 
Ivabradine $2 \mathrm{mg} / \mathrm{kg}$, i.v.
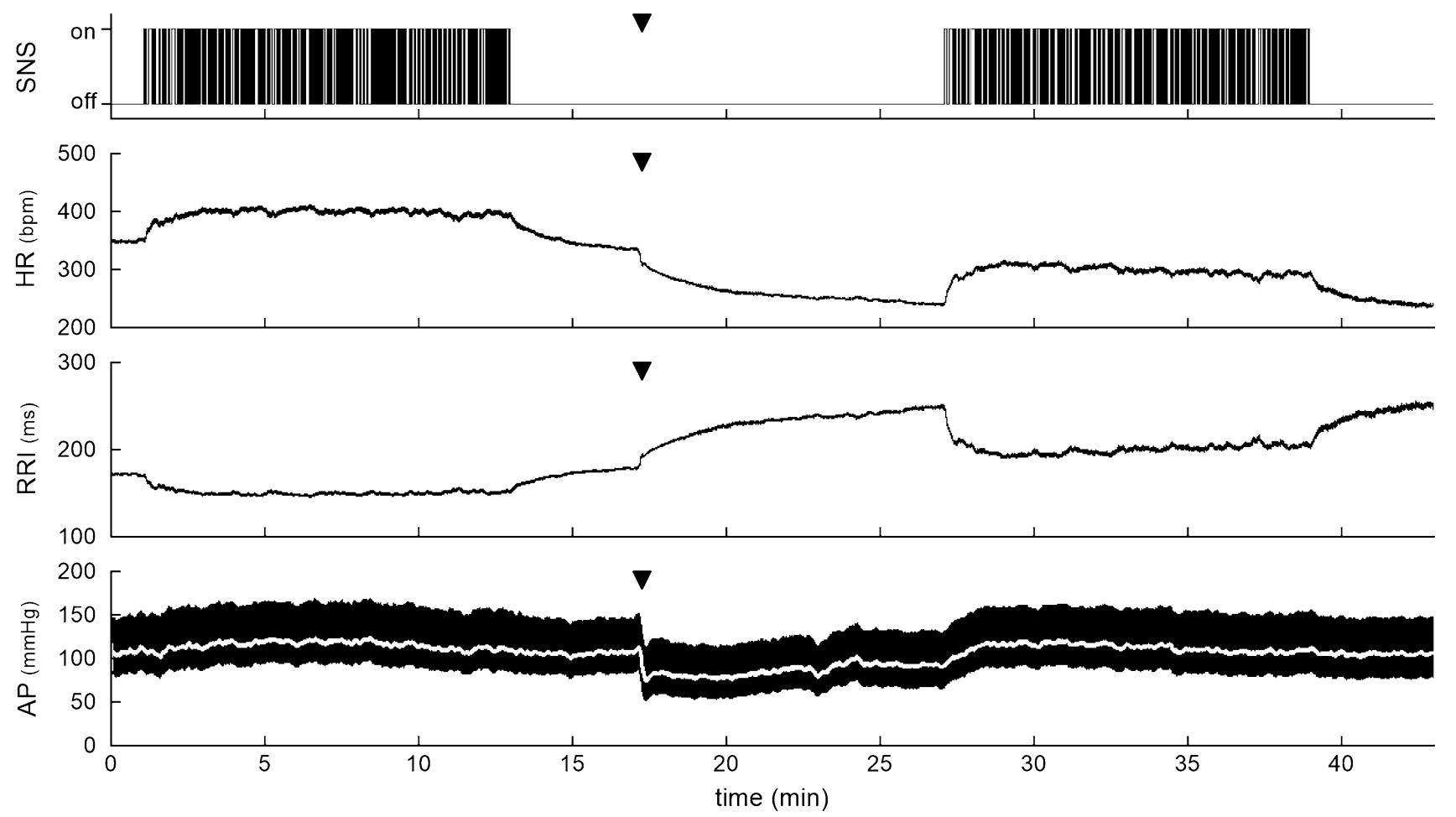

Fig. 1 Typical recordings of a sympathetic nerve stimulation (SNS) command, heart rate (HR), R-R interval (RRI), and arterial pressure (AP) obtained from the ivabradine protocol. The data are displayed as $200-\mathrm{Hz}$ resampled signals. The white line in the AP plot indicates a 2-s moving averaged signal. The first random SNS changed HR

\section{$-34.1 \pm 7.3 \mathrm{~ms}(P=0.019)$. Neither ivabradine nor SNS} significantly affected AP.

Figure 3 shows a typical transfer function analysis of the HR response to SNS. Figure 3a depicts a portion of the time series obtained during SNS before ivabradine. Random SNS dynamically changed HR. Figure $3 \mathrm{~b}$ illustrates the gain and phase plots of the transfer function from SNS to HR and the coherence function. The dynamic gain decreased with increasing frequency, indicating that the HR response to SNS has low-pass characteristics. The phase approached zero radians at the lowest frequency, reflecting a positive HR response to SNS during the steady state. The coherence was approximately 0.7 at the lowest frequency, increased to near unity in the frequency range from 0.03 to $0.3 \mathrm{~Hz}$, and decreased in the frequency range above $0.3 \mathrm{~Hz}$. Figure $3 \mathrm{c}$ shows a model transfer function fitted to the estimated transfer function. As depicted in Fig. 3d, the estimated and model transfer functions showed well-correlated gain and phase values. However, the estimated transfer function showed some dispersion in the gain range below 0.1 and the phase range below $-5 / 4 \pi$ radians, probably because the accuracy of the transfer function estimation was decreased in the highfrequency range due to low dynamic gain values. dynamically with an increase in the mean level of HR. Intravenous (i.v.) administration of ivabradine gradually decreased HR. The second random SNS also changed HR dynamically with an increase in the mean level of HR. RRI showed changes reciprocal to HR. bpm beats/min

Averaged transfer functions from SNS to HR, the coherence functions, and the corresponding step responses obtained from the ivabradine protocol are shown in Fig. 4a. The thin and bold lines indicate the transfer functions before (control) and after ivabradine, respectively. The gain and phase plots are nearly superimposed before and after ivabradine. Ivabradine tended to increase the asymptotic dynamic gain and decrease the natural frequency (Table 1). The damping ratio and the pure dead time did not change significantly. Ivabradine did not affect the coherence values that were averaged in the frequency range from 0.01 to $0.1 \mathrm{~Hz}$ (control: $0.79 \pm 0.05$ vs. ivabradine: $0.78 \pm 0.02, P=0.861$ by the bootstrap method), but significantly lowered the coherence values in the frequency range from 0.1 to $0.5 \mathrm{~Hz}$ (control: $0.42 \pm 0.06$ vs. ivabradine: $0.30 \pm 0.04, P=0.010$ by the bootstrap method). The step response showed an initial lag (pure delay) of approximately $1 \mathrm{~s}$ followed by a gradual increase of HR. Ivabradine did not affect the steadystate HR response or the $80 \%$ rise time (Table 1).

Averaged transfer functions from SNS to RRI, the coherence functions, and the corresponding step responses are shown in Fig. 4b. The dynamic gain decreased as the frequency increased, both before and after ivabradine. As a 
Fig. 2 Averaged data of heart rate (HR), R-R interval (RRI), and arterial pressure (AP) before (pre-SNS) and during sympathetic nerve stimulation (SNS) obtained from the ivabradine protocol. The open and filled bars indicate data obtained under control conditions and after the administration of ivabradine. The results of a two-way repeated-measure analysis of variance (ANOVA) are displayed. $\mathrm{bpm}$ beats/min. Data are mean and mean $+\mathrm{SE}$ values $(n=7)$

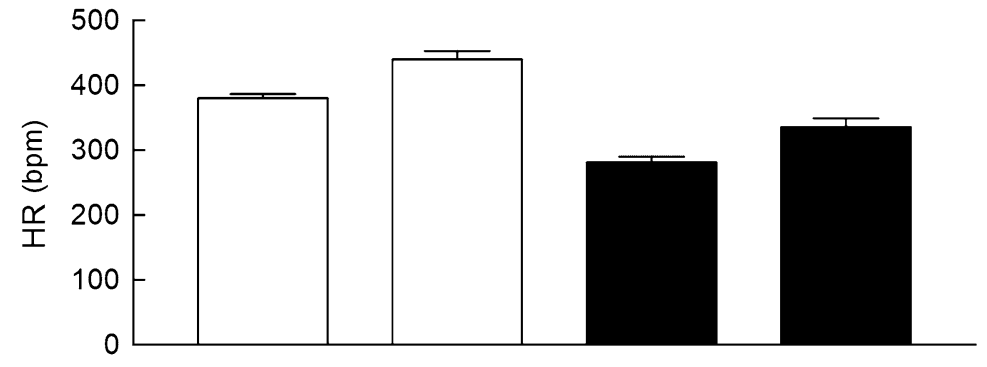

Two-way ANOVA Ivabradine: $P<0.001$ SNS: $\quad P<0.001$ Interaction: $P=0.820$

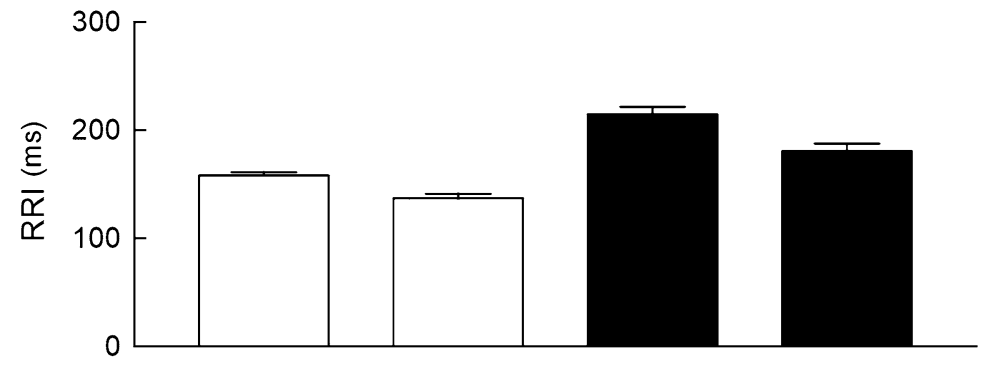

Two-way ANOVA Ivabradine: $P<0.001$ SNS: $\quad P<0.001$ Interaction: $P=0.127$

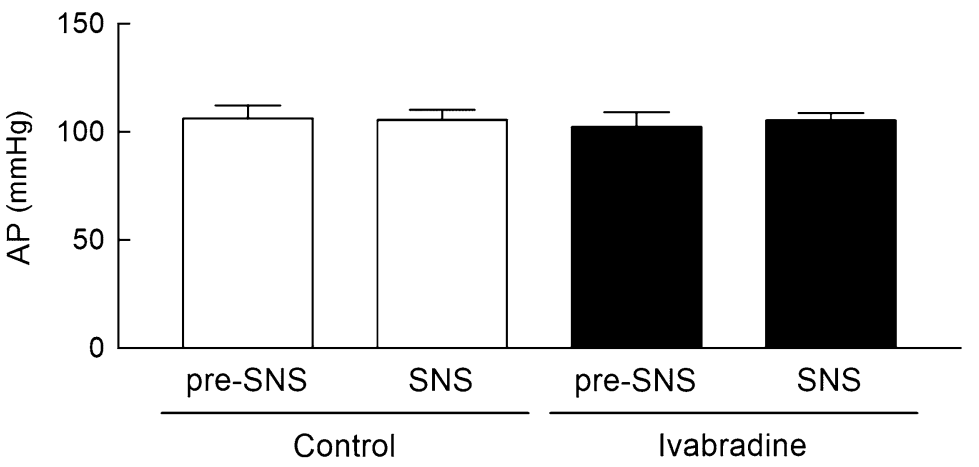

Two-way ANOVA Ivabradine: $P=0.547$ SNS: $\quad P=0.733$ Interaction: $P=0.577$ mathematical consequence, the gain plots of the RRI transfer function and the HR transfer function calculated from the same data are parallel vertically. Hence, the gain plots showing the mean RRI transfer function and the mean HR transfer function are only displaced along the ordinate, both before and after ivabradine (Fig. 4c). This does not mean, however, that the effects of ivabradine on the RRI and HR transfer functions are the same. Ivabradine significantly increased the asymptotic dynamic gain and tended to decrease the natural frequency (Table 1). The RRI and HR transfer functions were out of phase. The coherence function associated with the RRI transfer function was not different from that associated with the HR transfer function. Ivabradine nearly doubled the steady-state RRI response without affecting the $80 \%$ rise time (Table 1 ).

Typical entire recordings of SNS, HR, RRI, and AP, obtained from one animal in the metoprolol protocol, are shown in Fig. 5. The first random SNS increased mean HR and changed HR dynamically under the control conditions. Intravenous administration of metoprolol transiently decreased AP and acutely reduced HR. Ten minutes later, the second random SNS did not perceivably change HR. RRI changed in a manner reciprocal to HR. Averaged values of HR, RRI, and AP are illustrated in Fig. 6. The effects of metoprolol and SNS on HR were statistically significant with a significant interaction effect, suggesting that the effect of SNS differed between before and after metoprolol. Metoprolol attenuated SNS-induced increase in the mean HR from $87.5 \pm 13.7$ to $6.5 \pm 1.9 \mathrm{bpm}(P=0.002)$. The effects of metoprolol and SNS on RRI were also statistically significant, and further exhibited a significant interaction effect. Metoprolol attenuated SNS-induced decrease in the mean RRI from $-28.7 \pm 4.2$ to $-3.3 \pm 1.0 \mathrm{~ms}(P=0.001)$. Metoprolol tended to increase mean AP, whereas SNS did not significantly affect the mean AP.

The change of the pre-stimulation HR induced by metoprolol ( $-42.6 \pm 5.5 \mathrm{bpm})$ was significantly less negative than that induced by ivabradine $(-98.8 \pm 5.3 \mathrm{bpm}, P<0.001)$. The change of the pre-stimulation RRI induced by metoprolol $(20.8 \pm 3.2 \mathrm{~ms})$ was significantly smaller than that induced by ivabradine $(56.5 \pm 4.9 \mathrm{~ms}, P<0.001)$.

Averaged transfer functions from SNS to HR, the coherence functions, and the corresponding step responses observed during the metoprolol protocol are shown in Fig. 7a. The thin and bold lines indicate the transfer functions before (control) and after metoprolol, respectively. Metoprolol significantly reduced dynamic gain values and rendered the transfer function more variable among animals. 
a
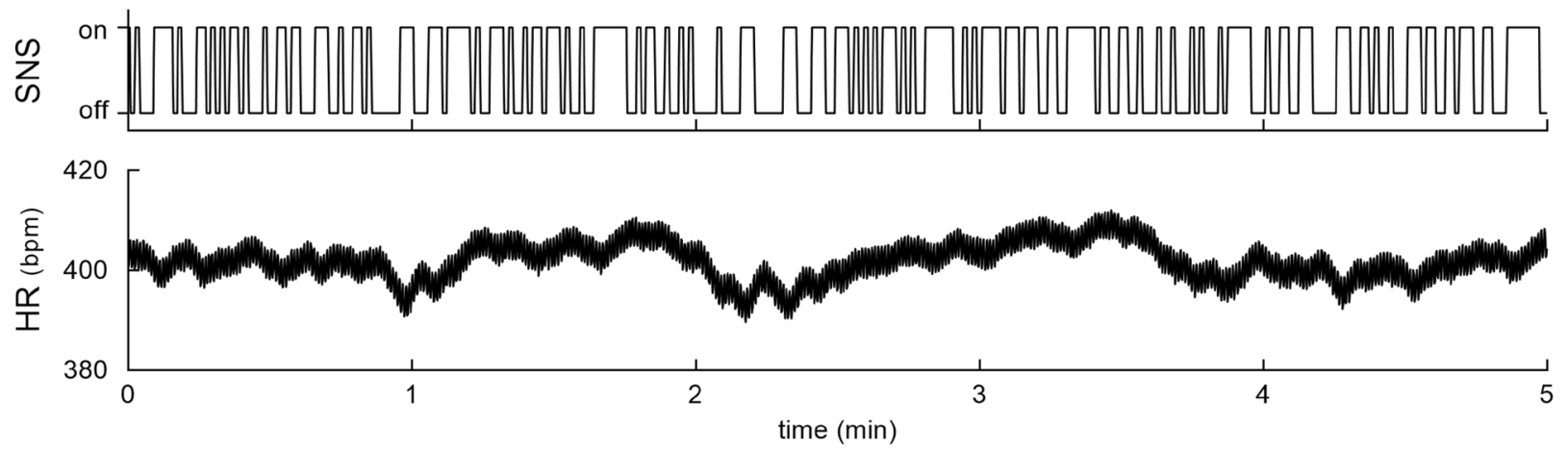

b
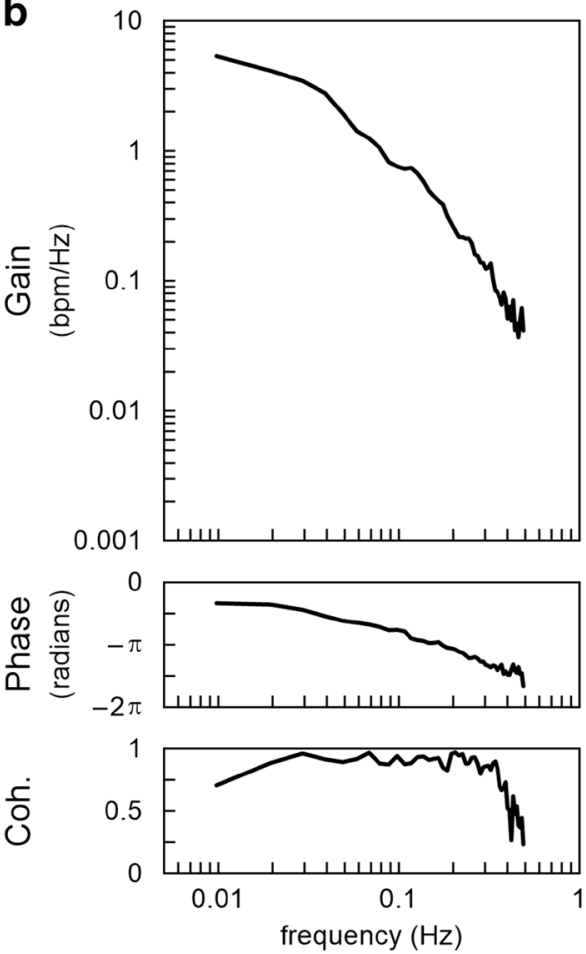

C 10
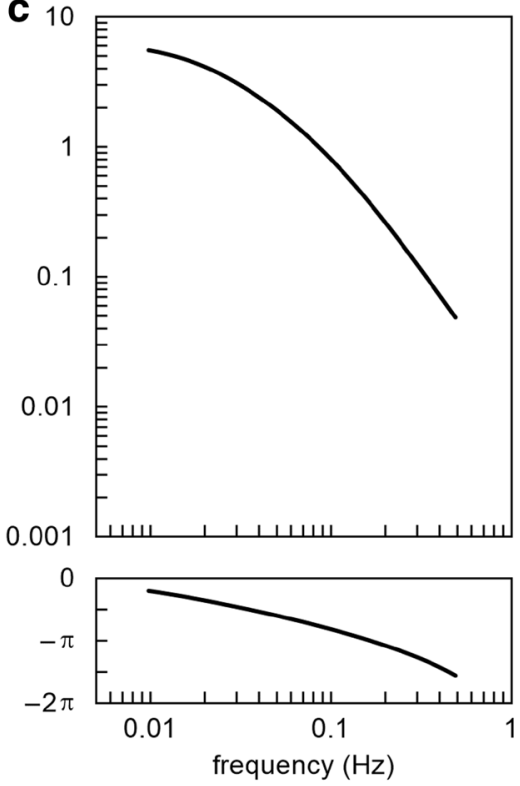
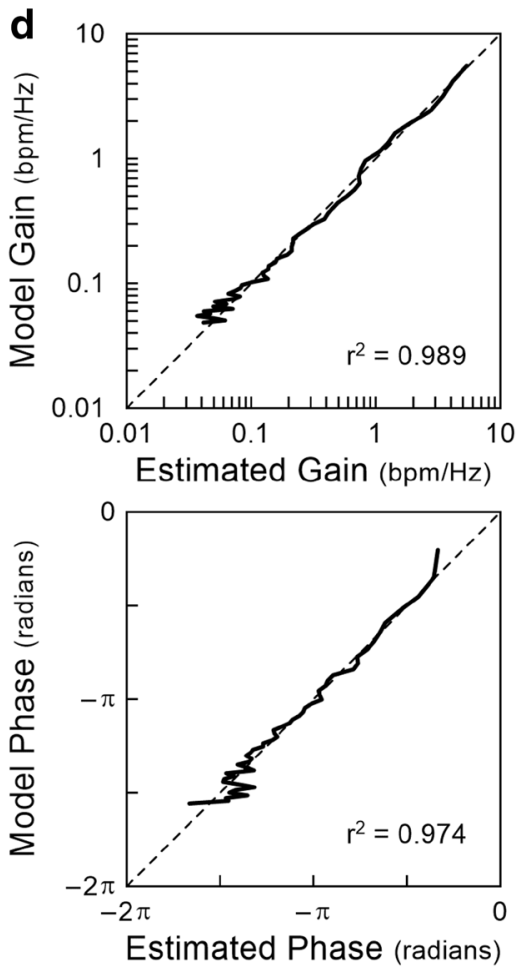

Fig. 3 a A portion of Fig. 1 showing a sympathetic nerve stimulation (SNS) command and the heart rate (HR) response. The data are displayed as $10-\mathrm{Hz}$ resampled signals. $\mathrm{bpm}$ beats/min. b A transfer function from SNS to HR. The gain and phase plots and the coherence (Coh.) associated with the transfer function are shown. c A model

The dynamic gain at the lowest frequency $\left(G_{0.01}\right)$ decreased from $4.58 \pm 0.38$ to $0.42 \pm 0.11 \mathrm{bpm} / \mathrm{Hz}(P<0.001)$. Metoprolol significantly reduced the coherence values averaged in the frequency range from 0.01 to $0.1 \mathrm{~Hz}$ (control: $0.86 \pm 0.02$ vs. metoprolol: $0.52 \pm 0.11, P<0.001$ by the bootstrap method) and in the frequency range from 0.1 to $0.5 \mathrm{~Hz}$ (control: $0.45 \pm 0.03$ vs. metoprolol: $0.17 \pm 0.03, P<0.001$ by the bootstrap method). The step response was negligibly small after metoprolol compared with the pre-metoprolol response. The steady-state HR response $\left(S_{50}\right)$ decreased from $5.23 \pm 0.41$ to $0.24 \pm 0.13 \mathrm{bpm} / \mathrm{Hz}(P<0.001)$. The RRI transfer function and step response (Fig. 7b) changed in a manner similar to the HR transfer function and step transfer function (Eq. 3) fitted to the estimated transfer function. d Plots showing the gain and phase of the model transfer function versus those of the estimated transfer function. The model transfer function described the estimated transfer function with reasonable accuracy. The dotted lines indicate the line of identity

response [i.e., metoprolol decreased $G_{0.01}$ from $1.30 \pm 0.15$ to $0.19 \pm 0.05 \mathrm{~ms} / \mathrm{Hz}(P=0.001)$ and attenuated $S_{50}$ from $-1.46 \pm 0.15$ to $-0.12 \pm 0.06 \mathrm{~ms} / \mathrm{Hz}(P<0.001)]$. The gain plots of the mean RRI transfer function and the mean HR transfer function are parallel vertically, both before and after metoprolol (Fig. 7c).

\section{Discussion}

Although ivabradine induces bradycardia by inhibiting HCN channels, its effect on dynamic sympathetic control of HR has never been elucidated. HCN channels are an essential 
a
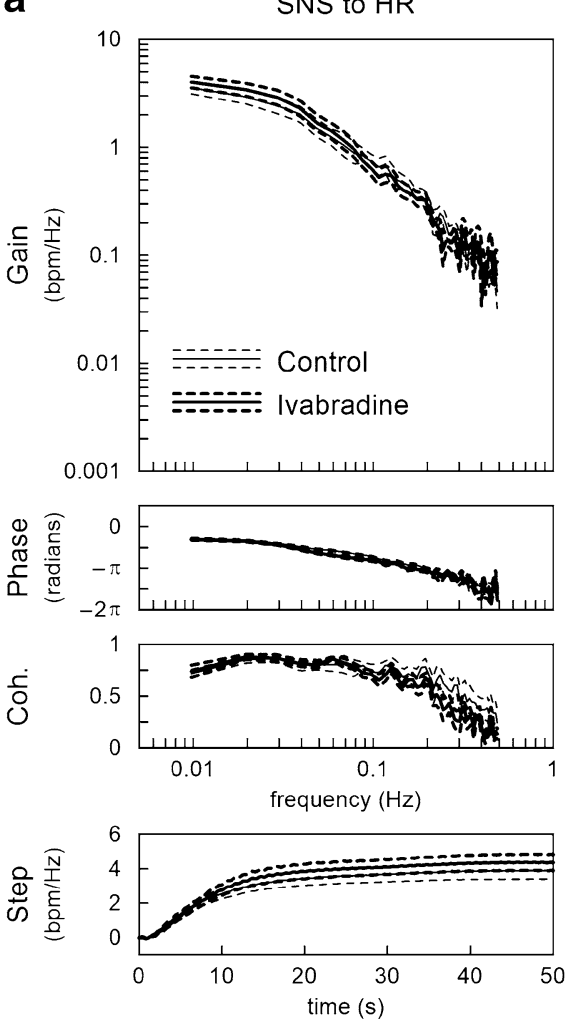

b
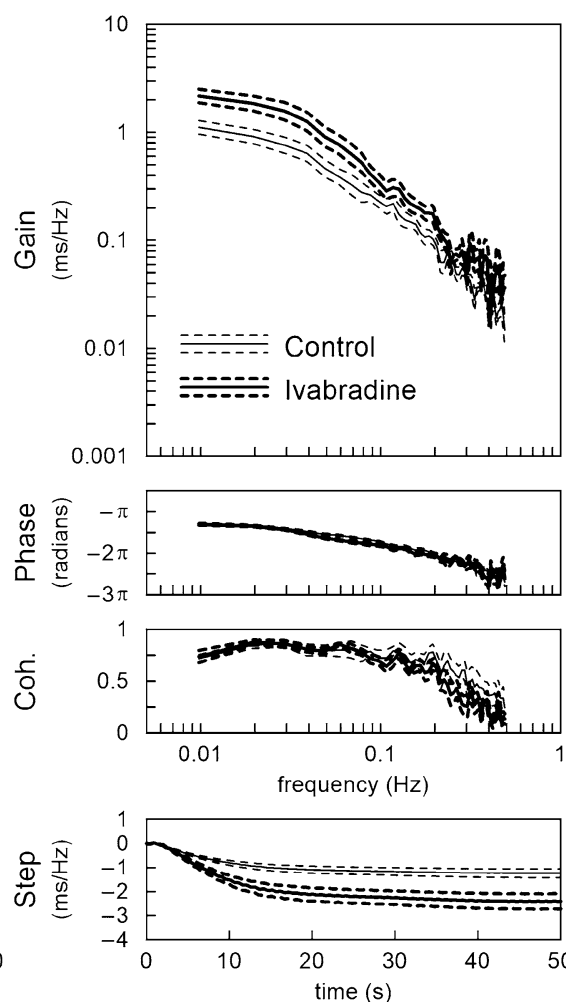

C
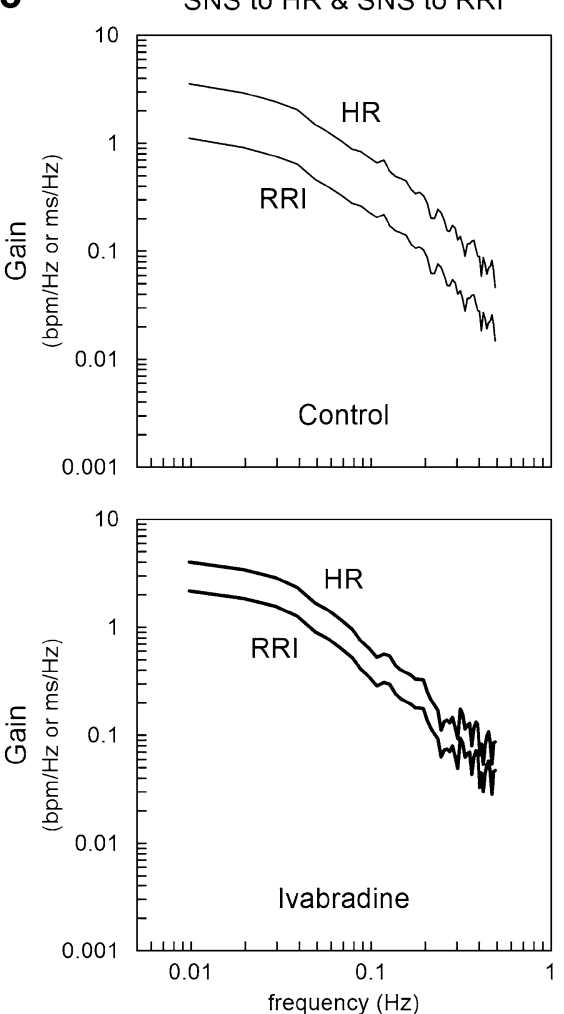

Fig. 4 a Averaged transfer functions from sympathetic nerve stimulation (SNS) to heart rate (HR), coherence functions (Coh.), and the corresponding step responses before (thin lines) and after (bold lines) the administration of ivabradine. All plots, excepting the coherence in the frequency range above approximately $0.2 \mathrm{~Hz}$, were not significantly different between before and after ivabradine. b Averaged transfer functions from SNS to R-R interval (RRI), coherence function, and the corresponding step response before (thin lines) and after (bold lines) the administration of ivabradine. Ivabradine increased dynamic gain of the RRI transfer function and augmented the negative step response of RRI. c The mean transfer functions calculated for the HR response and the RRI response are only displaced along the ordinate in the gain plots, both before (top panel) and after (bottom panel) ivabradine. bpm beats/min. The solid and dashed lines in panels $\mathbf{a}$ and $\mathbf{b}$ indicate mean and mean \pm SE values $(n=7)$ component mediating the positive chronotropic response to SNS [3]. Nevertheless, we found that intravenous ivabradine did not attenuate the dynamic sympathetic control of HR, despite inducing significant bradycardia. The dynamic gain could be even augmented after ivabradine when the response was analyzed by RRI. By contrast, metoprolol abolished the dynamic sympathetic control of HR.

\section{Effects of ivabradine on dynamic HR and RRI responses to SNS}

As mentioned in the introduction, $\mathrm{HCN}$ channels are a key component for mediating the positive chronotropic response to SNS. Among four $\mathrm{HCN}$ isoforms known, HCN4 is the most highly expressed isoform in the sinoatrial nodal cells [3]. Inhibiting $\mathrm{HCN}$ channels by ivabradine could interfere with the HR response to SNS. In our previous study using vagotomized rats, however, intravenous ivabradine did not reduce the magnitude of the $\mathrm{HR}$ response to static pressure changes in carotid sinus baroreceptors, suggesting that ivabradine spares the sympathetic control of HR despite inducing significant bradycardia [6]. In the present study, we focused on the dynamic aspect of the sympathetic control of HR by using random SNS. Ivabradine did not attenuate the dynamic gain of the HR transfer function and even augmented the dynamic gain of the RRI transfer function (Fig. 4, Table 1). Hence, ivabradine preserved the dynamic sympathetic control of HR.

The coherence function in the frequency range above $0.1 \mathrm{~Hz}$ was lower after ivabradine than before ivabradine, suggesting some interference of ivabradine on the linearity in the sympathetic control of HR. In our previous study, ivabradine does not increase atrial fibrillation inducibility, irrespective of underlying tonic vagal activity in dogs [20]. On the other hand, ivabradine does not protect from atrial arrhythmias during experimental transition from cardiac sympathetic activation to vagal predominance in humans [21]. Although the vagal nerves were not involved in the 
Table $1 \mathrm{HR}$ and RRI transfer function and step response parameters obtained from ivabradine protocol

\begin{tabular}{|c|c|c|c|}
\hline & Control & Ivabradine & $P$ value \\
\hline \multicolumn{4}{|c|}{ HR transfer function } \\
\hline$K, \mathrm{bpm} / \mathrm{Hz}$ & $4.32 \pm 0.56$ & $4.93 \pm 0.55$ & 0.077 \\
\hline$f_{\mathrm{N}}, \mathrm{Hz}$ & $0.066 \pm 0.005$ & $0.054 \pm 0.004$ & 0.072 \\
\hline$\zeta$ & $1.75 \pm 0.18$ & $1.59 \pm 0.22$ & 0.222 \\
\hline$L, \mathrm{~s}$ & $0.77 \pm 0.05$ & $0.84 \pm 0.07$ & 0.434 \\
\hline \multicolumn{4}{|c|}{ HR step response } \\
\hline$S_{50}, \mathrm{bpm} / \mathrm{Hz}$ & $3.89 \pm 0.49$ & $4.37 \pm 0.46$ & 0.510 \\
\hline$T_{80 \%}, \mathrm{~s}$ & $14.1 \pm 0.9$ & $14.8 \pm 1.0$ & 0.720 \\
\hline \multicolumn{4}{|c|}{ RRI transfer function } \\
\hline$K, \mathrm{~ms} / \mathrm{Hz}$ & $1.38 \pm 0.23$ & $2.64 \pm 0.32^{* *}$ & $<0.001$ \\
\hline$f_{\mathrm{N}}, \mathrm{Hz}$ & $0.066 \pm 0.005$ & $0.054 \pm 0.005$ & 0.071 \\
\hline$\zeta$ & $1.75 \pm 0.19$ & $1.54 \pm 0.23$ & 0.272 \\
\hline$L, \mathrm{~s}$ & $0.77 \pm 0.05$ & $0.85 \pm 0.07$ & 0.428 \\
\hline \multicolumn{4}{|c|}{ RRI step response } \\
\hline$S_{50}, \mathrm{~ms} / \mathrm{Hz}$ & $-1.23 \pm 0.18$ & $-2.40 \pm 0.31^{*}$ & 0.012 \\
\hline$T_{80 \%}, \mathrm{~s}$ & $14.1 \pm 0.9$ & $14.6 \pm 1.0$ & 0.767 \\
\hline
\end{tabular}

Data are mean \pm SE values $(n=7)$

$H R$ heart rate, $b p m$ beats/min, $R R I \mathrm{R}-\mathrm{R}$ interval, $K$ asymptotic dynamic gain, $f_{\mathrm{N}}$ natural frequency, $\zeta$ damping ratio, $L$ pure dead time, $S_{50}$ steady-state step response at $50 \mathrm{~s}, T_{80 \%} 80 \%$-rise time of the step response. $P$ values were determined by paired $t$ tests

*** $P<0.05$ and $P<0.01$, respectively

present study due to vagotomy, on/off transitions during the random SNS might have induced atrial premature activity after ivabradine, which could reduce the linearity between SNS and the HR response.

The increase in the dynamic gain of the RRI transfer function after ivabradine is consistent with the finding of the augmented response range of RRI during static pressure changes in carotid sinus baroreceptors in our previous study [6]. Action potentials of cardiac cells are characterized by a rapid depolarization (phase 0), a rapid reversal of the overshoot potential (phase 1), a long plateau (phase 2), and a repolarization (phase 3) to a stable resting membrane potential (phase 4) [22]. The pacemaker cells exhibit a slowly depolarizing resting potential during phase 4 . We have postulated that the sympathetic effect on RRI may be enhanced when ivabradine reduces the rate of the depolarization during phase 4. As an example, if we assume that SNS doubles the $I_{\mathrm{f}}$ current by acting on HCN channels without affecting the other parts of the action potential, the duration of phase 4 would be approximately halved. When the duration of phase 4 is $80 \mathrm{~ms}$ under control conditions, SNS will shorten the duration of phase 4 to $40 \mathrm{~ms}$ (the RRI shortening by $40 \mathrm{~ms}$ ). Next, let us assume that ivabradine inhibits HCN channels and prolongs the duration of phase 4 to $160 \mathrm{~ms}$. SNS will still double the $I_{\mathrm{f}}$ current by acting on $\mathrm{HCN}$ channels that are not blocked by ivabradine, shortening the duration of phase
4 to $80 \mathrm{~ms}$ (the RRI shortening by $80 \mathrm{~ms}$ ). Hence, the effect of SNS on the RRI could be enhanced when the number of available HCN channels was reduced by ivabradine [6]. Changes in HR are not parallel with changes in RRI because they are inversely related. In addition, changes in HR depend on the duration other than phase 4 in a cardiac cycle. If we assume that the duration other than phase 4 is $90 \mathrm{~ms}$, the baseline HR is calculated to be $60,000 /(90+80)=353 \mathrm{bpm}$, which increases to $60,000 /(90+40)=462 \mathrm{bpm}$ during the SNS (the HR increase by $109 \mathrm{bpm}$ ). After ivabradine, the pre-stimulation HR is $60,000 /(90+160)=240 \mathrm{bpm}$, which increases to $60,000 /(90+80)=353 \mathrm{bpm}$ during the SNS (the HR increase by $113 \mathrm{bpm}$ ). Hence, ivabradine will double the gain for the RRI response to SNS (80/40) whereas only slightly increase the gain for the HR response to SNS $(113 / 109)$ in this numerical consideration.

Apparently, the above interpretation is overly simplistic, and further considerations are required regarding the effects of cAMP on HR independent of HCN channels. For instance, Alig et al. [23] generated mice with heart-specific and inducible expression of a human $\mathrm{HCN} 4$ mutation that abolishes the cAMP-dependent regulation of HCN channels. The mice showed a marked reduction in HR at rest and during exercise. However, the sinoatrial nodal cells of the mice maintained the relative responsiveness to a $\beta_{1}$-adrenergic agonist isoproterenol, suggesting that the sensitivity of $\mathrm{HCN}$ channels to cAMP is not a prerequisite for the sympathetic HR control. Subjects who have an HCN mutation with subunits insensitive to cAMP demonstrate full ability to accelerate HR [24]. Further studies with the quantification of the cAMP generation during SNS before and after ivabradine are required to identify the mechanisms regarding how ivabradine preserves the sympathetic HR control despite inducing significant bradycardia.

Although ivabradine tended to decrease the natural frequency of the HR transfer function, it did not significantly affect the $80 \%$ rise time of the HR step response, suggesting that ivabradine exerted little effect on the HR response speed (Table 1). By contrast, a blockade of neuronal norepinephrine uptake by intravenous desipramine reduced the natural frequency of the HR transfer function and delayed the HR response to SNS [8]. This suggests that norepinephrine disposition at the sinoatrial node neuroeffector junction may be a rate-limiting step of the dynamic HR response to SNS. The release of norepinephrine from sympathetic nerve terminals is modulated by presynaptic autoinhibition, mediated by $\alpha_{2}$ adrenergic receptors. A blockade of $\alpha_{2}$-adrenergic receptors by intravenous yohimbine reduced the natural frequency and increased the asymptotic dynamic gain of the HR transfer function, suggesting that the norepinephrine release can also affect the HR response speed [25]. It is likely that intracellular processes, from $\beta_{1}$-adrenergic stimulation to changes in the rate of diastolic depolarization, are faster than the 


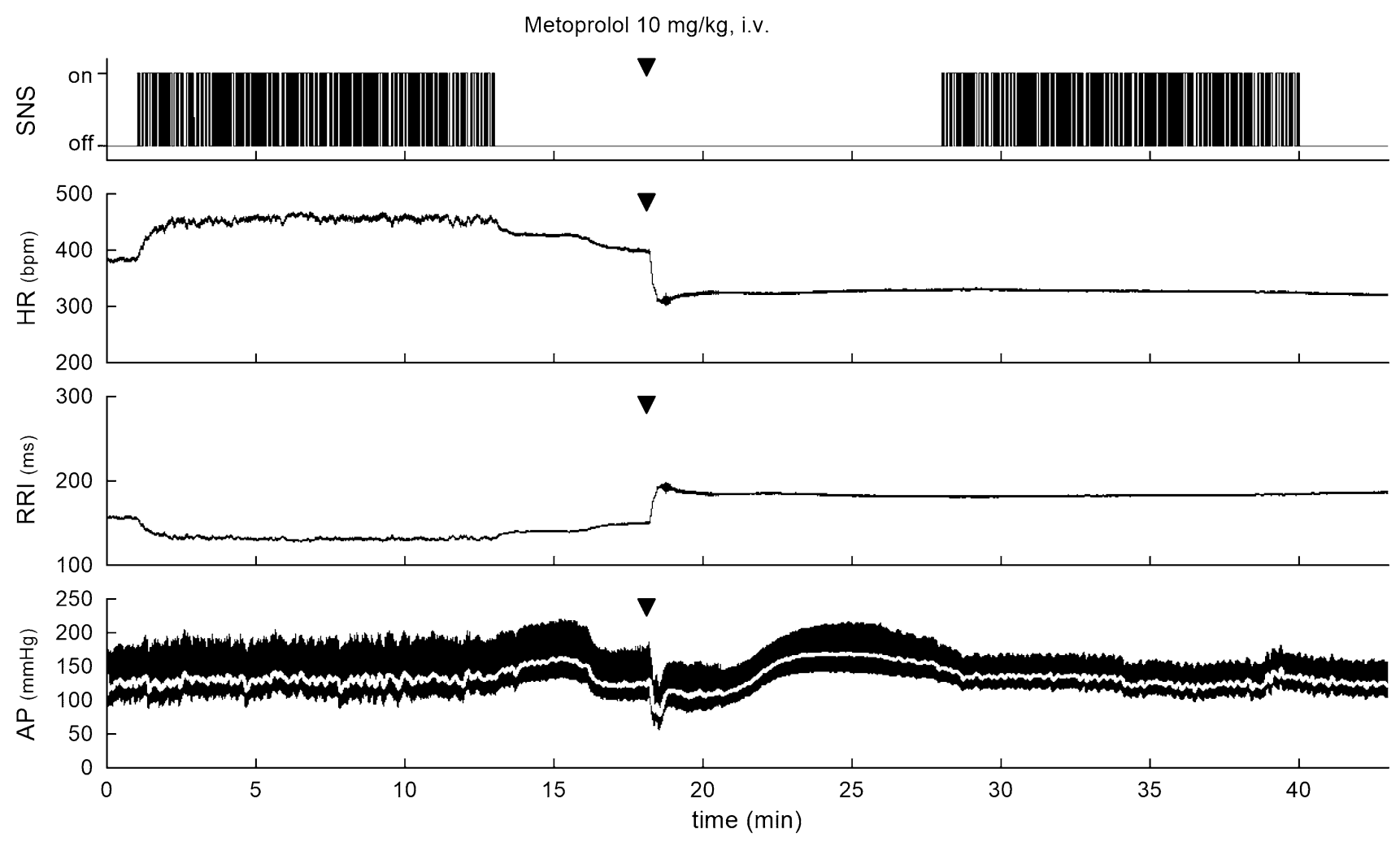

Fig. 5 Typical recordings of a sympathetic nerve stimulation (SNS) command, heart rate (HR), R-R interval (RRI), and arterial pressure (AP) obtained from the metoprolol protocol. The data are displayed as $200-\mathrm{Hz}$ resampled signals. The first random SNS changed HR

extracellular processes of norepinephrine release and disposition; therefore, the blockade of HCN channels by ivabradine did not significantly affect the response speed of HR to SNS.

\section{Effects of metoprolol on dynamic HR and RRI responses to SNS}

Although we sectioned bilateral cervical sympathetic nerves, other sympathetic nerves directed to the heart, such as those originating from the thoracic spinal cord, were kept intact. Metoprolol blocked the sympathetic drive to the heart and decreased HR. The bradycardic effect (i.e., the reduction of pre-stimulation HR) induced by metoprolol was approximately half that induced by ivabradine. This was not due to an insufficient dose of metoprolol because metoprolol nearly abolished an increase in the mean HR during SNS (Fig. 6). Despite the smaller magnitude of the bradycardic effect compared with ivabradine, metoprolol significantly reduced the dynamic gains of the HR and RRI transfer functions. The process of system identification using the white noise input was robust enough to capture some residual low-pass characteristics of the HR and RRI transfer functions even after dynamically with an increase in the mean level of HR. Intravenous (i.v.) administration of metoprolol acutely decreased HR. The second random SNS did not change HR perceivably. RRI showed changes reciprocal to HR. bpm beats/min

metoprolol. However, the HR and RRI responses to SNS reduced to less than $1 / 10$, as can be seen in the corresponding step responses (Fig. 7a, b, bottom panels). Hence, metoprolol, at the dose used in the present study, abolished the dynamic sympathetic control of HR even though the bradycardic effect was smaller than that induced by ivabradine.

The intracellular concentration of cAMP may be able to increase in response to SNS after ivabradine, contributing to an increase in HR. In contrast, metoprolol blocks the binding of norepinephrine to $\beta_{1}$-adrenergic receptors, making the intracellular concentration of cAMP unresponsive to SNS. The lessor bradycardic effect of metoprolol compared with ivabradine indicates that the basal sympathetic tone may not play a substantial role in maintaining the basal HR under normal physiological conditions. On the other hand, HCN channels could be a primary determinant of the basal HR, though a different view exists in which the $I_{f}$ current is regarded as a safety net preventing excess bradycardia [26].

\section{Limitations}

There are several limitations to the present study. First, because ivabradine or metoprolol were administered 
Fig. 6 Averaged data of heart rate (HR), R-R interval (RRI), and arterial pressure (AP) before (pre-SNS) and during sympathetic nerve stimulation (SNS) obtained from the metoprolol protocol. The open and filled bars indicate data obtained under control conditions and after the administration of metoprolol. The results of a two-way repeated-measure analysis of variance (ANOVA) are displayed. bpm beats/min. Data are mean and mean + SE values $(n=6)$

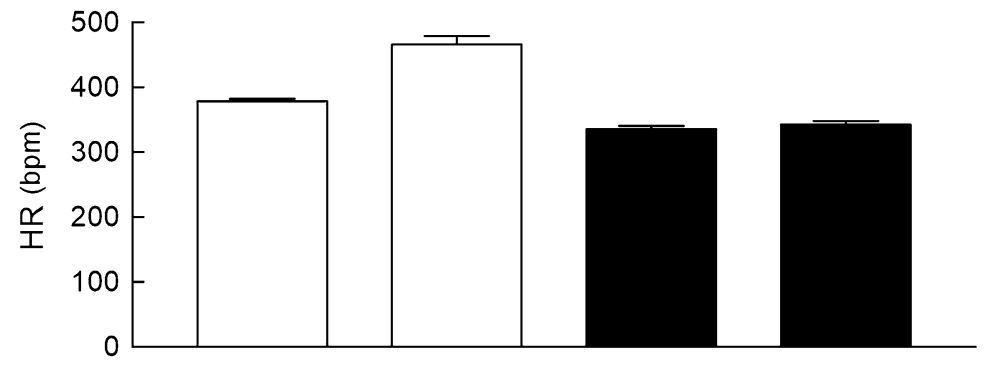

Two-way ANOVA Metoprolol: $P<0.001$ SNS: $\quad P<0.001$ Interaction: $P<0.001$

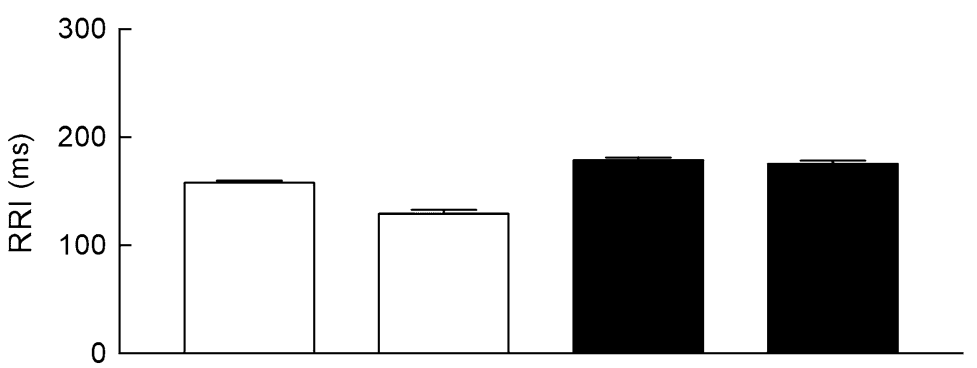

Two-way ANOVA Metoprolol: $P<0.001$ SNS: $\quad P<0.001$ Interaction: $P<0.001$

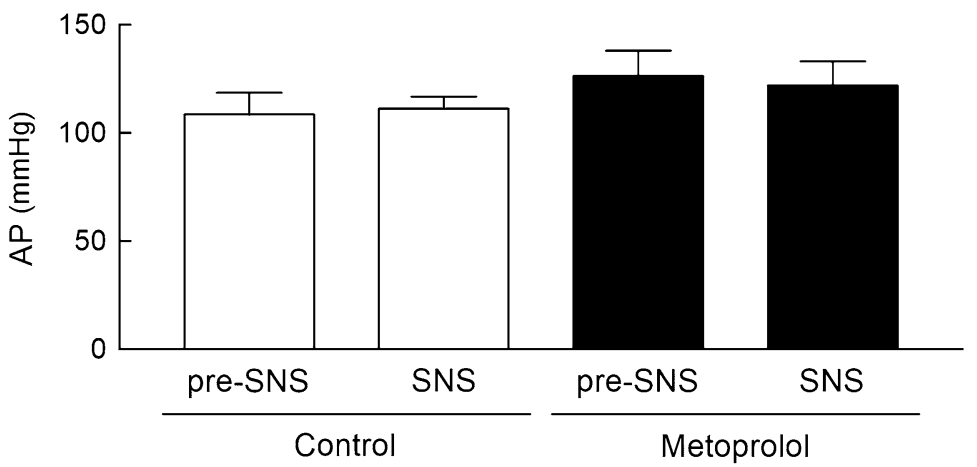

Two-way ANOVA Metoprolol: $P=0.058$ SNS: $\quad P=0.903$ Interaction: $P=0.615$ acutely under anesthesia, the results cannot directly account for any chronic effects of the drug, administered under conscious conditions. Second, the experiment was performed on normal rats. The results could be different had we performed the experiment using diseased models such as chronic heart failure after myocardial infarction. Third, we stimulated the cervical sympathetic nerve rather than the cardiac sympathetic nerve due to technical difficulties. The majority of the stimulated nerve was preganglionic because an intravenous administration of hexamethonium $(60 \mathrm{mg} / \mathrm{kg})$ nearly abolished the HR response to the cervical SNS (data not shown). Although ivabradine does not reach the central nervous system due to the blood-brain barrier, it could affect ganglionic transmission by modifying the hyperpolarization-activated current $\left(I_{\mathrm{h}}\right)$ in neuronal cells, which is also generated by $\mathrm{HCN}$ channels [27].

In conclusion, intravenous ivabradine did not attenuate the dynamic sympathetic control of HR despite inducing significant bradycardia. The preserved dynamic sympathetic control of HR with coexisting bradycardia is one of the unique characteristics that could not be achieved by a $\beta$-blocker, and may contribute to some beneficial effects of ivabradine reported in clinical application. 
a
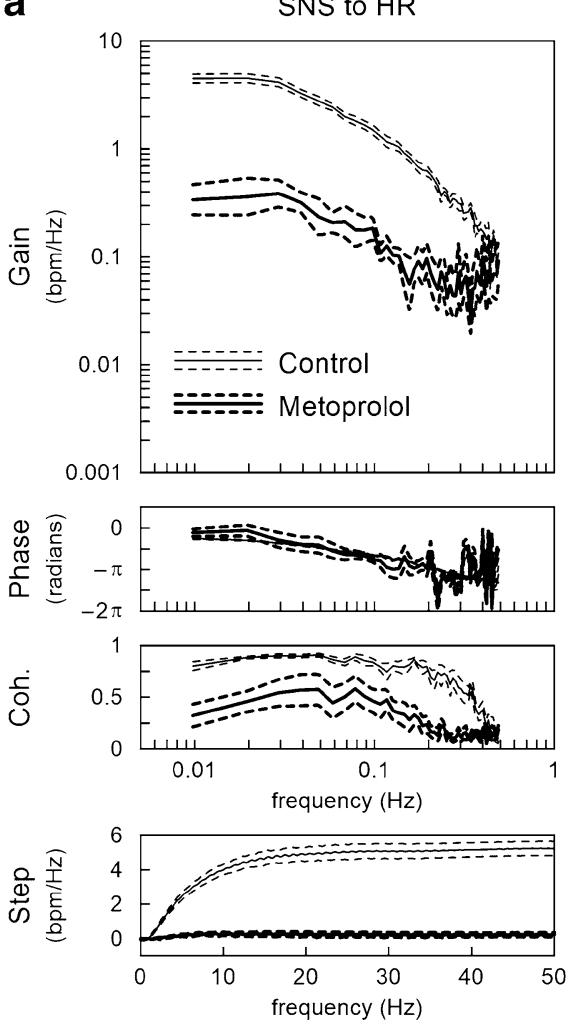

b
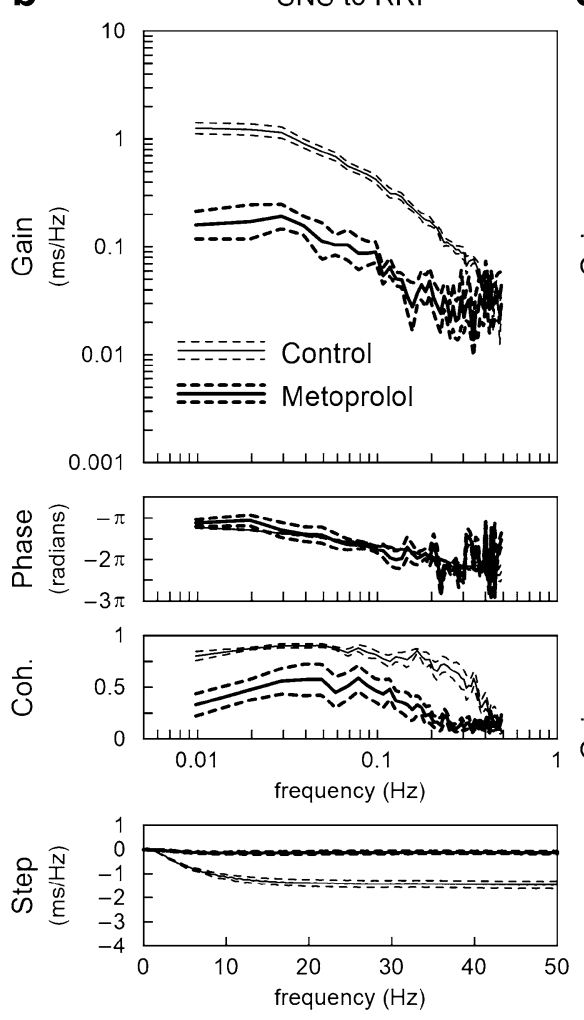

C
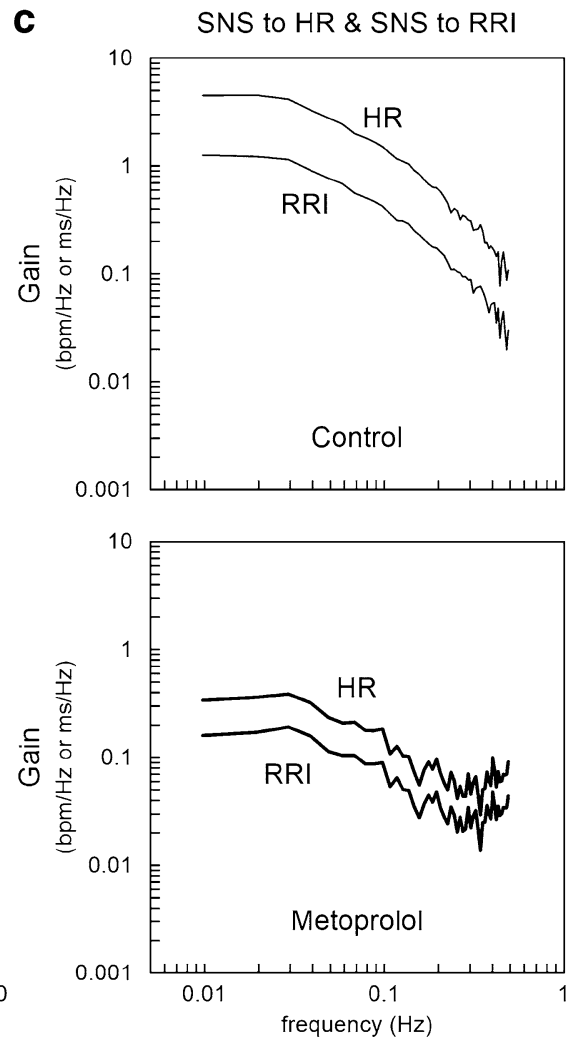

Fig. 7 a Averaged transfer functions from sympathetic nerve stimulation (SNS) to heart rate (HR), coherence functions (Coh.), and the corresponding step responses before (thin lines) and after (bold lines) the administration of metoprolol. Metoprolol reduced the dynamic gain and disrupted the HR transfer function. Coherence was lower after metoprolol compared with the control condition. The step response was nearly absent after metoprolol. b Averaged transfer functions from SNS to R-R interval (RRI), coherence function, and the corresponding step response before (thin lines) and after

Author contributions TK and MS, conception and design of the study; TK performed the experiment and analyzed the data; TK, SS, KU, YH, HY, TS, TN, and MS, discussed and interpreted the results. TK and MS, drafting and revising the manuscript. All authors have read the final version of the manuscript and approved its submission to the Journal of Physiological Sciences.

\section{Compliance with ethical standards}

Research involving human participants and/or animals Animals used in the present study were cared for in strict accordance with the Guiding Principles for the Care and Use of Animals in the Field of Physiological Sciences, which has been approved by the Physiological Society of Japan. The Animal Subjects Committee at the National Cerebral and Cardiovascular Center reviewed and approved the experimental protocols.

Informed consent Not applicable.

Funding This study was supported in part by a Grant-in-Aid for Scientific Research (JSPS KAKENHI Grant number 18K10695). (bold lines) the administration of metoprolol. Metoprolol decreased the dynamic gain and disrupted the RRI transfer function. Metoprolol abolished the negative step response of RRI. $\mathbf{c}$ The mean transfer functions calculated for the HR response and the RRI response are only displaced along the ordinate in the gain plots, both before (top panel) and after (bottom panel) metoprolol. bpm beats/min. The solid and dashed lines in panels $\mathbf{a}$ and $\mathbf{b}$ indicate mean and mean \pm SE values $(n=6)$

Conflict of interest The authors declare that there are no conflicts of interest.

\section{References}

1. Vinogradova TM, Lyashkov AE, Zhu W, Ruknudin AM, Sirenko S, Yang D, Deo S, Barlow M, Johnson S, Caffrey JL, Zhou YY, Xiao RP, Cheng H, Stern MD, Maltsev VA, Lakatta EG (2006) High basal protein kinase A-dependent phosphorylation drives rhythmic internal $\mathrm{Ca}^{2+}$ store oscillations and spontaneous beating of cardiac pacemaker cells. Circ Res 98:505-514

2. Liao Z, Lockhead D, Larson ED, Proenza C (2010) Phosphorylation and modulation of hyperpolarization-activated HCN4 channels by protein kinase A in the mouse sinoatrial node. J Gen Physiol 136:247-258

3. DiFrancesco D, Borer JS (2007) The funny current: cellular basis for the control of heart rate. Drugs 67(Suppl 2):15-24

4. Hjalmarson $\AA$ (2007) Heart rate: an independent risk factor in cardiovascular disease. Eur Heart J Suppl 9(Suppl F):F3-F7

5. Swedberg K, Komajda M, Böhm M, Borer JS, Ford I, DubostBrama A, Lerebours G, Tavazzi L, SHIFT Investigators (2010) 
Ivabradine and outcomes in chronic heart failure (SHIFT): a randomised placebo-controlled study. Lancet 376:875-885

6. Yamamoto H, Kawada T, Shimizu S, Uemura K, Inagaki M, Kakehi K, Iwanaga Y, Fukuda K, Miyamoto T, Miyazaki S, Sugimachi M (2018) Ivabradine does not acutely affect open-loop baroreflex static characteristics and spares sympathetic heart rate control in rats. Int J Cardiol 257:255-261

7. Heusser K, Tank J, Brinkmann J, Schroeder C, May M, Großhennig A, Wenzel D, Diedrich A, Sweep FC, Mehling H, Luft FC, Jordan J (2016) Preserved autonomic cardiovascular regulation with cardiac pacemaker inhibition: a crossover trial using highfidelity cardiovascular phenotyping. J Am Heart Assoc 5:e002674

8. Nakahara T, Kawada T, Sugimachi M, Miyano H, Sato T, Shishido T, Yoshimura R, Miyashita H, Inagaki M, Alexander J Jr, Sunagawa K (1999) Neuronal uptake affects dynamic characteristics of heart rate response to sympathetic stimulation. Am J Physiol 277:R140-R146

9. Berger RD, Saul JP, Cohen RJ (1989) Transfer function analysis of autonomic regulation. I. Canine atrial rate response. Am J Physiol 256:H142-H152

10. Kawada T, Ikeda Y, Sugimachi M, Shishido T, Kawaguchi O, Yamazaki T, Alexander J Jr, Sunagawa K (1996) Bidirectional augmentation of heart rate regulation by autonomic nervous system in rabbits. Am J Physiol 271:H288-H295

11. Mizuno M, Kawada T, Kamiya A, Miyamoto T, Shimizu S, Shishido T, Smith SA, Sugimachi M (2010) Dynamic characteristics of heart rate control by the autonomic nervous system in rats. Exp Physiol 95:919-925

12. Shoukas AA, Callahan CA, Lash JM, Haase EB (1991) New technique to completely isolate carotid sinus baroreceptor regions in rats. Am J Physiol 260:H300-H303

13. Sato T, Kawada T, Miyano H, Shishido T, Inagaki M, Yoshimura R, Tatewaki T, Sugimachi M, Alexander J Jr, Sunagawa K (1999) New simple methods for isolating baroreceptor regions of carotid sinus and aortic depressor nerves in rats. Am J Physiol 276:H326-H332

14. Dias da Silva VJ, Tobaldini E, Rocchetti M, Wu MA, Malfatto G, Montano N, Zaza A (2015) Modulation of sympathetic activity and heart rate variability by ivabradine. Cardiovasc Res 108:31-38

15. Gardiner SM, Kemp PA, March JE, Bennett T (1995) Acute and chronic cardiac and regional haemodynamic effects of the novel bradycardic agent, S16257, in conscious rats. Br J Pharmacol 115:579-586
16. Di Verniero CA, Silberman EA, Mayer MA, Opezzo JA, Taira CA, Höcht $C$ (2008) In vitro and in vivo pharmacodynamic properties of metoprolol in fructose-fed hypertensive rats. J Cardiovasc Pharmacol 51:532-541

17. Bendat JS, Piersol AG (2010) Random data. Analysis and measurement procedures, 4th edn. Wiley, New Jersey

18. Glantz SA, Slinker BK (2001) Primer of applied regression and analysis of variance, 2nd edn. McGraw-Hill, New York

19. Efron B, Tibshirani RJ (1994) An introduction to the bootstrap. Chapman \& Hall, New York

20. Uemura K, Inagaki M, Zheng C, Kawada T, Li M, Fukumitsu M, Sugimachi M (2017) Acute ivabradine treatment reduces heart rate without increasing atrial fibrillation inducibility irrespective of underlying vagal activity in dogs. Heart Vessels 32:484-494

21. Chobanyan-Jürgens K, Heusser K, Duncker D, Veltmann C, May M, Mehling H, Luft FC, Schröder C, Jordan J, Tank J (2018) Cardiac pacemaker channel (HCN4) inhibition and atrial arrhythmogenesis after releasing cardiac sympathetic activation. Sci Rep $8: 7748$

22. Mohrman DE, Heller LJ (2010) Cardiovascular physiology 7th edn. A LANGE medical book. McGraw Hill, New York, pp 24-25

23. Alig J, Marger L, Mesirca P, Ehmke H, Mangoni ME, Isbrandt D (2009) Control of heart rate by cAMP sensitivity of HCN channels. Proc Natl Acad Sci USA 106:12189-12194

24. Schweizer PA, Duhme N, Thomas D, Becker R, Zehelein J, Draguhn A, Katus HA, Koenen M (2010) cAMP sensitivity of HCN pacemaker channels determines basal heart rate but is not critical for autonomic rate control. Circ Arrhythm Electrophysiol 3:542-552

25. Miyamoto T, Kawada T, Yanagiya Y, Akiyama T, Kamiya A, Mizuno M, Takaki H, Sunagawa K, Sugimachi N (2008) Contrasting effects of presynaptic $\alpha_{2}$-adrenergic autoinhibition and pharmacologic augmentation of presynaptic inhibition on sympathetic heart rate control. Am J Physiol Heart Circ Physiol 295:H1855-H1866

26. Monfredi O, Maltsev VA, Lakatta EG (2013) Modern concepts concerning the origin of the heartbeat. Physiology (Bethesda) 28:74-92

27. Doan TN, Stephans K, Ramirez AN, Glazebrook PA, Andresen MC, Kunze DL (2004) Differential distribution and function of hyperpolarization-activated channels in sensory neurons and mechanosensitive fibers. J Neurosci 24:3335-3343 